PROCEEDINGS OF THE

AMERICAN MATHEMATICAL SOCIETY

Volume 125, Number 12, December 1997, Pages 3679-3687

S 0002-9939(97)04123-3

\title{
INVARIANCE OF THE $L_{p}$ SPECTRUM FOR HYPOELLIPTIC OPERATORS
}

\author{
HANS-GERD LEOPOLD AND ELMAR SCHROHE
}

(Communicated by Palle E. T. Jorgensen)

\begin{abstract}
We show that the spectra of the $L_{p}$-realizations for a class of hypoelliptic (pseudo-)differential operators are independent of $p$ in an interval around $p=2$ depending on the growth properties of the symbol. For elliptic operators we obtain the classical boundedness interval of Fefferman; in the general case we obtain a smaller interval which is as large as one can possibly expect it to be.
\end{abstract}

\section{INTRODUCTION}

Every differential operator $A$ on Euclidean space naturally induces an unbounded operator $A_{p}$ on $L_{p}\left(\mathbb{R}^{n}\right)$. The question to what extent its spectrum depends on $p$ is of considerable interest both in the theory of partial differential equations and mathematical physics.

This problem attracted additional attention when B. Simon conjectured that the spectrum of a Schrödinger operator $-\Delta+V$ is independent of $p$. Following upon work by Simon and Sigal, Hempel and Voigt [9] showed in 1986 that the conjecture holds for a rather general class of potentials $V$.

Their result has since been extended in various directions. On the other hand, one also knows many situations where the spectrum does depend on $p$. For several different aspects see e.g. Arendt [2], Davies [4], [5], Davies, Simon, and Taylor [6], Sturm [18].

Hieber [10] recently considered Fourier multipliers on $\mathbb{R}^{n}$. Under a hypoellipticity assumption he was able to prove that the $L_{p}$-spectrum of each such operator agrees with its $L_{2}$-spectrum for all $p$ in a certain interval around $p=2$. Through his paper we became convinced that it should be possible to bring in pseudodifferential techniques.

In fact, the problem how the spectrum varies with $p$ also has been considered in connection with algebras of zero-order singular integral and pseudodifferential operators. Here, too, one encounters both phenomena in the literature: There are early results by Widom [20] and Gohberg-Krupnik [8] on $p$-dependence, while the

Received by the editors July 29, 1996.

1991 Mathematics Subject Classification. Primary 35P05, 35H05, 47G30.

Key words and phrases. $L_{p}$-spectrum, spectral independence, hypoelliptic pseudodifferential operators.

The first author was supported in part by DFG-contract $\operatorname{Tr} 374 / 1-1$.

(C) 1997 American Mathematical Society 
articles Alvarez-Hounie [1], Leopold-Schrohe [14], and Schrohe [16] suggest invariance to a very large extent. Although the question of invertibility is of a quite different type in the bounded context, some of the methods still apply.

In the present article we show that the spectrum of a hypoelliptic (pseudo-) differential operator is independent of $p$ in an interval around $p=2$. Moreover, this interval is as large as we can realistically expect it to be.

The idea of our proof is rather simple: We show that the $L_{p}$-resolvent is a pseudodifferential operator and therefore furnishes an inverse on all spaces where it is continuous. Apart from a mild technical condition, we assume that the operator $A$ is given by a symbol $a$ of order $m>0$ in Hörmander's class $S_{\rho, \delta}^{m}$ for $0 \leq \delta<\rho \leq 1$, and that it satisfies a hypoellipticity condition which allows us to construct a parametrix with symbol in $S_{\rho, \delta}^{-m^{\prime}}, m^{\prime}>0$. Applying a theorem by Fefferman [7] we see that the parametrix will only extend to a bounded operator on $L_{p}\left(\mathbb{R}^{n}\right)$, if $\left|\frac{1}{2}-\frac{1}{p}\right| n(1-\rho) \leq m^{\prime}$. This is precisely the interval where we establish $p$-invariance. In fact there is a classical counterexample to boundedness if this condition is violated, namely the Fourier multiplier of Hardy-Littlewood-HirschmannWainger; see [7]. It is given by op $\left(\exp \left(i[\xi]^{1-\rho}\right) /[\xi]^{m^{\prime}}\right)$, where $[\cdot]$ is a smooth positive function on $\mathbb{R}^{n}$ with $[\xi]=|\xi|$ for large $|\xi|$. Its symbol is an element in $S_{\rho, 0}^{-m^{\prime}}$ yet the operator is unbounded on $L_{p}\left(\mathbb{R}^{n}\right)$ whenever $p$ falls outside the above range. In the elliptic case, i.e., for $m=m^{\prime}$, this shows the maximality of the asserted interval.

Concerning $L_{p^{-}}$-spectra, $1<p<\infty$, our result both extends and improves that of [10]: For one thing, we cover the case of $x$-dependent symbols; for another, we get a larger interval of independence if $m^{\prime}<m$.

A certain draw-back of the pseudodifferential methods is that they require $C^{\infty}$ smoothness. It is rather obvious that this assumption can be relaxed; it is much more difficult, however, to say to what extent this is true. This will be the subject of a forthcoming publication. It seems to be a new point, however, that we can guarantee an interval of $p$-independence in terms of symbol estimates, without requiring self-adjointness, Gaussian estimates, or uniform ellipticity.

\section{REsults}

Let $m \geq m^{\prime}>0,0 \leq \delta<\rho \leq 1$, and let $a: \mathbb{R}^{n} \times \mathbb{R}^{n} \rightarrow \mathbb{C}$ be a smooth $\left(C^{\infty}\right)$ complex-valued function satisfying the following two conditions:

(i) $\left|D_{\xi}^{\alpha} D_{x}^{\beta} a(x, \xi)\right| \leq C_{\alpha \beta}\langle\xi\rangle^{m-\rho|\alpha|+\delta|\beta|}$; i.e., $a$ is an element of $S_{\rho, \delta}^{m}=S_{\rho, \delta}^{m}\left(\mathbb{R}^{2 n}\right)$.

(ii) The argument of $a(x, \xi)$ is a bounded continuous function, there is an $R>0$ such that $a(x, \xi)$ is invertible for $|\xi| \geq R$, and

$$
\begin{aligned}
\left|D_{\xi}^{\alpha} D_{x}^{\beta} a(x, \xi) a^{-1}(x, \xi)\right| & \leq C_{\alpha \beta}\langle\xi\rangle^{-\rho|\alpha|+\delta|\beta|}, \\
\left|a^{-1}(x, \xi)\right| & \leq C\langle\xi\rangle^{-m^{\prime}}, \quad|\xi| \geq R .
\end{aligned}
$$

The symbol $a$ defines the pseudodifferential operator op $a: \mathcal{S} \rightarrow \mathcal{S}$ by means of the formula

$$
[\text { op } a] u(x)=\int e^{i x \xi} a(x, \xi) \hat{u}(\xi) d \xi
$$

As usual, $\mathcal{S}=\mathcal{S}\left(\mathbb{R}^{n}\right)$ is the Schwartz space of rapidly decreasing functions, $\hat{u}(\xi)=$ $\int e^{-i x \xi} u(x) d x$ is the Fourier transform of $u$, and $d \xi=(2 \pi)^{-n} d \xi$. 


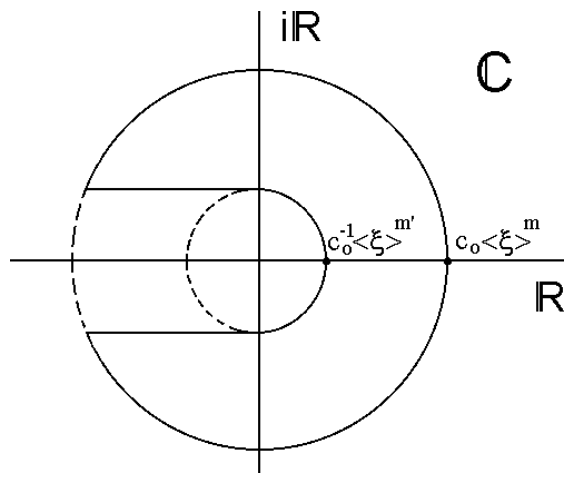

Figure 1 . The set $\Omega_{0}$

The symbol $a$ may also take values in the space of $l \times l$-matrices over $\mathbb{C}$. In this case, we consider $u$ in (1.1) as an $l$-vector of functions in $\mathcal{S}$. For simplicity we shall write $\mathcal{S}, L_{p}, H_{p}^{s}, C_{0}^{\infty}$, etc. without referring to either $\mathbb{R}^{n}$ or $l$. In the matrix-valued case we replace (ii) by the following condition:

(ii') There exist a bounded, real-valued continuous function $\theta=\theta(x, \xi)$ and an $R>0$ such that all eigenvalues of $a(x, \xi)$ are in the domain $\Omega_{x, \xi}$ defined below, and

$$
\left|D_{\xi}^{\alpha} D_{x}^{\beta} a(x, \xi) \circ(a(x, \xi)-\zeta)^{-1}\right| \leq C_{\alpha \beta}\langle\xi\rangle^{-\rho|\alpha|+\delta|\beta|},|\xi| \geq R, \zeta \in \mathbb{C} \backslash \Omega_{x, \xi} .
$$

The choice of the domains $\Omega_{x, \xi}$ goes back to Kumano-go [12, Chapter 8]. One lets $\Omega_{x, \xi}=e^{i \theta(x, \xi)} \Omega_{0}$ where, for $m \geq m^{\prime}>0$ and some fixed $c_{0}>1$,

$$
\Omega_{0}=\left\{z \in \mathbb{C}: c_{0}^{-1}\langle\xi\rangle^{m^{\prime}}<|z|<c_{0}\langle\xi\rangle^{m} \text {, and }|\operatorname{Im} z|>c_{0}^{-1}\langle\xi\rangle^{m^{\prime}} \text { for } \operatorname{Re} z<0\right\} \text {. }
$$

In particular it follows from (ii') that $\left|a(x, \xi)^{-1}\right| \leq C\langle\xi\rangle^{-m^{\prime}}$.

For $1<p<\infty$ we let $A_{p}$ denote the $L_{p}$-realization of $A=$ op $a$ with $D\left(A_{p}\right)=$ $\left\{u \in L_{p}: A u \in L_{p}\right\}$. Notice that $A_{p}$ is a closed operator. The resolvent set of $A_{p}$ is written $\rho\left(A_{p}\right)$, the spectrum $\sigma\left(A_{p}\right)$.

We shall prove the following theorem.

Theorem 1.1. Given a symbol a with properties (i) and (ii) or (i) and (ii'), the spectrum of the operators $A_{p}$ is the same for all $1<p<\infty$ with $m^{\prime} \geq n\left|\frac{1}{2}-\frac{1}{p}\right|(1-\rho)$.

Remark 1.2. (a) For $\rho=1$, all $1<p<\infty$ are admissible, since symbols in $S_{1, \delta}^{0}$ define bounded operators on $L_{p}$, cf. Illner [11]. In this case, the additional condition on the existence of a continuous argument function (or its analog in the matrix case) is not necessary; see Lemma 1.3 below.

(b) For $\rho<1$, the condition $m^{\prime} \geq n\left|\frac{1}{2}-\frac{1}{p}\right|(1-\rho)$ guarantees the boundedness of operators with symbols in $S_{\rho, \delta}^{-m^{\prime}}$ on $L_{p}$, see Fefferman [7].

Lemma 1.3. Let $A=$ op $a$ with an elliptic $a \in S_{1, \delta}^{m}, 0 \leq \delta<1, m \geq 0$. Then the spectrum of $A_{p}$ is independent of $p$ for $1<p<\infty$.

Ellipticity means that there is an $R>0$ such that $a(x, \xi)$ is both invertible whenever $|\xi| \geq R$ and satisfies the estimate $a(x, \xi)^{-1}=O\left(\langle\xi\rangle^{-m}\right)$. 


\section{Proofs}

Let us start with the simplest case.

2.1. Proof of Lemma 1.3. The ellipticity of $a$ implies that $D\left(A_{p}-\lambda\right)=H_{p}^{m}$, the $L_{p}$-based Sobolev space of order $m$. Invertibility of $A_{p}-\lambda$ as an unbounded operator on $L_{p}$ therefore is the same as invertiblity of the pseudodifferential operator $(A-\lambda) \circ$ op $\langle\xi\rangle^{-m}$ as a bounded operator on $L_{p}$. It is well-known that the latter is independent of $p$ for $1<p<\infty$, see e.g. [16, Corollary 1.9].

The proof of Theorem 1.1 is more difficult; it is therefore split up into a series of propositions and lemmata. We write $\Psi_{\rho, \delta}^{\mu}$ for the space of all operators $B: \mathcal{S} \rightarrow \mathcal{S}$ that can be written as a pseudodifferential operator in the form (1.1) with a symbol $b \in S_{\rho, \delta}^{\mu}, \mu \in \mathbb{R}$. The space $\Psi^{-\infty}=\bigcap_{\mu \in \mathbb{R}} \Psi_{\rho, \delta}^{\mu}$ is easily seen to be independent of the choice of $\rho$ and $\delta$.

Lemma 2.2. For every $\lambda \in \mathbb{C}$ there is a parametrix $B_{\lambda}=$ op $b_{\lambda} \in \Psi_{\rho, \delta}^{-m^{\prime}}$ to $A-\lambda$ with

$$
B_{\lambda}(A-\lambda)=I+R_{1}, \quad(A-\lambda) B_{\lambda}=I+R_{2}
$$

and $R_{1}, R_{2} \in \Psi^{-\infty}$. We understand (2.1) as an identity for operators on $\mathcal{S}$.

Proof. First let $a$ be scalar. For every fixed $\lambda$, the symbol $a(x, \xi)-\lambda$ of $A-\lambda$ satisfies conditions (i) and (ii). We obtain the assertion from [12, Chapter 2, Theorem 5.4]. For the matrix-valued case we apply Kumano-go [12, Chapter 8, Theorem 1.2]; see also Remark 2.8 and Theorem 2.9 below.

Lemma 2.3. For $\lambda \in \rho\left(A_{p}\right)$ and $\varphi \in \mathcal{S}$,

$$
\left(A_{p}-\lambda\right)^{-1} \varphi=B_{\lambda} \varphi-B_{\lambda} R_{2} \varphi+R_{1}\left(A_{p}-\lambda\right)^{-1} R_{2} \varphi \text { in } \mathcal{S}^{\prime} .
$$

Here, $B_{\lambda}, R_{1}$, and $R_{2}$ are the pseudodifferential operators from Lemma 2.2.

Proof. This is immediate since every one of these pseudodifferential operators is continuous both on $\mathcal{S}$ and $\mathcal{S}^{\prime}$, under the assumption $\delta<1$.

2.4. Outline. According to the composition rules for pseudodifferential operators, $B_{\lambda} R_{2}$ is an element of $\Psi^{-\infty}$. We are now going to show that

$$
R_{1}\left(A_{p}-\lambda\right)^{-1} R_{2} \in \Psi^{-\infty} \text {. }
$$

Then, in view of Lemma 2.3, we conclude that the restriction $\left.\left(A_{p}-\lambda\right)^{-1}\right|_{\mathcal{S}}$ coincides with a pseudodifferential operator in $\Psi_{\rho, \delta}^{-m^{\prime}}$, say $C$. By Fefferman's theorem [7] the operator $C$ extends to a bounded operator on $L_{q}$ for all $q$ with $1<q<\infty$ and $m^{\prime} \geq n\left|\frac{1}{2}-\frac{1}{q}\right|(1-\rho)$. Denote this extension by $C_{q}$. The identity

$$
\left(A_{p}-\lambda\right)\left(A_{p}-\lambda\right)^{-1}=I \quad \text { on } \quad L_{p}
$$

shows that $(A-\lambda) C \varphi=\varphi$ for all $\varphi \in \mathcal{S}$ since $C: \mathcal{S} \rightarrow \mathcal{S}$ is continuous. As $C_{q}$ is closed, $C_{q}$ maps $L_{q}$ to $D\left(A_{q}\right)$, and

$$
\left(A_{q}-\lambda\right) C_{q}=I \quad \text { on } \quad L_{q} .
$$

Similarly, $A-\lambda$ maps $\mathcal{S}$ to $\mathcal{S}$ and $C(A-\lambda)=I$ on $\mathcal{S}$. We conclude that

$$
C_{q}\left(A_{q}-\lambda\right) u=u \text { for all } u \in D\left(A_{q}\right) .
$$

Hence $C_{q}=\left(A_{q}-\lambda\right)^{-1}$, so $\lambda \in \rho\left(A_{q}\right)$. 
It therefore remains to show (2.2). In order to do this we shall employ the following well-known theorem. For a proof see e.g. Ueberberg [19] or Schrohe [17].

Theorem 2.5. A scalar linear operator $T: \mathcal{S} \rightarrow \mathcal{S}^{\prime}$ is a regularizing pseudodifferential operator if and only if for all multi-indices $\alpha, \beta$, and all $s, t \in \mathbb{R}$, the iterated commutator $\operatorname{ad}^{\alpha} x \operatorname{ad}^{\beta} D_{x}(T)$ extends to a bounded operator

$$
\operatorname{ad}^{\alpha} x \operatorname{ad}^{\beta} D_{x} T: H_{p}^{s} \rightarrow H_{p}^{t} \text {, for some } p \text { with } 1<p<\infty \text {. }
$$

If $T$ is an $l \times l$-matrix of linear operators, then $T$ is regularizing if and only if (2.3) holds for each component. Equivalently we may ask the boundedness of

$$
\operatorname{ad}^{\alpha} x I \operatorname{ad}^{\beta} D_{x} I(T): H_{p}^{s} \rightarrow H_{p}^{t}
$$

Here $I$ is the $l \times l$ identity matrix. Recall the ad-notation: For a multi-index $\beta=$ $\left(\beta_{1}, \ldots, \beta_{n}\right)$ one lets $\operatorname{ad}^{\beta} D_{x}(T)=\operatorname{ad}^{\beta_{1}} D_{x_{1}} \ldots \operatorname{ad}^{\beta_{n}} D_{x_{n}}(T)$ with $\operatorname{ad}^{0} D_{x_{j}}(T)=T$ and $\operatorname{ad}^{k} D_{x_{j}}(T)=\left[D_{x_{j}}, \operatorname{ad}^{k-1} D_{x_{j}}(T)\right], k \geq 1$. Analogously we define $\operatorname{ad}^{\alpha} x(T)$. The notation extends to expressions of the form $\operatorname{ad}^{k} S(T)$ for operators $S$ acting on $\mathcal{S}$ or $\mathcal{S}^{\prime}$ provided that all compositions make sense. Leibniz' rule states that

$$
\operatorname{ad}^{k} S\left(T_{1} T_{2}\right)=\sum_{k_{1}+k_{2}=k} c_{k_{1} k_{2}} \operatorname{ad}^{k_{1}} S\left(T_{1}\right) \operatorname{ad}^{k_{2}} S\left(T_{2}\right) .
$$

For a pseudodifferential operator $T=$ op $t$ one has

$$
\operatorname{ad} x_{j}(T)=-\operatorname{op}\left(D_{\xi_{j}} t\right) \quad \text { and } \quad \operatorname{ad} D_{x_{j}}(T)=\text { op } D_{x_{j}} t .
$$

2.6. Reduction. We want to show that, for all multi-indices $\alpha, \beta$, and all $s, t$,

$$
\operatorname{ad}^{\alpha} x \operatorname{ad}^{\beta} D_{x}\left(R_{1}\left(A_{p}-\lambda\right)^{-1} R_{2}\right): H_{p}^{s} \rightarrow H_{p}^{t} \text { is bounded. }
$$

The definition shows that $\operatorname{ad}^{\beta} D_{x}\left(R_{1}\left(A_{p}-\lambda\right)^{-1} R_{2}\right)$ is a linear combination of terms of the form $R_{1}^{\prime}\left(A_{p}-\lambda\right)^{-1} R_{2}^{\prime}$ with $R_{1}^{\prime}, R_{2}^{\prime} \in \Psi^{-\infty}$. Hence it is no restriction to assume $\beta=0$. On the other hand, by Leibniz' rule and (2.4) it is sufficient to consider operators of the form

$$
R_{1}^{\prime \prime} \operatorname{ad}^{\alpha} x\left(A_{p}-\lambda\right)^{-1} R_{2}^{\prime \prime}
$$

and to show that $\operatorname{ad}^{\alpha} x\left(A_{p}-\lambda\right)^{-1}$ is bounded in $L_{p}$. In order to do this we shall first consider a simple commutator $\left[x,\left(A_{p}-\lambda\right)^{-1}\right]$ where $x$ now stands for any of the matrix functions $x_{1} I, \ldots, x_{n} I$. In the case $m \leq \rho$ it will be easy to show our result; see Lemma 2.7. For $m>\rho$ we shall use complex powers to reformulate $A_{p}-\lambda$ as a composition of lower order operators.

Lemma 2.7. Assume additionally that $m \leq \rho$. Then $[x, A] \in \Psi_{\rho, \delta}^{0}$, and

$$
\begin{aligned}
{\left[x,\left(A_{p}-\lambda\right)^{-1}\right] } & =-\left(A_{p}-\lambda\right)^{-1}[x,(A-\lambda)]\left(A_{p}-\lambda\right)^{-1} \\
& =-\left(A_{p}-\lambda\right)^{-1}[x, A]\left(A_{p}-\lambda\right)^{-1} \\
& =-B_{\lambda}[x, A]\left(A_{p}-\lambda\right)^{-1}+\left(A_{p}-\lambda\right)^{-1} R_{2}[x, A]\left(A_{p}-\lambda\right)^{-1}
\end{aligned}
$$

in $\mathcal{L}\left(\mathcal{S}, \mathcal{S}^{\prime}\right)$. The right-hand side furnishes an extension to an operator in $\mathcal{L}\left(L_{p}\right)$, since $\left(A_{p}-\lambda\right)^{-1} \in \mathcal{L}\left(L_{p}\right)$ and $B_{\lambda}[x, A], R_{2}[x, A] \in \Psi_{\rho, \delta}^{-m^{\prime}}$ induce operators in $\mathcal{L}\left(L_{p}\right)$.

For $m \leq \rho$, induction therefore shows relation (2.5), and the proof of Theorem 1.1 is complete. 
Proof. Choose a function $\varphi \in C_{0}^{\infty}$ with $\varphi(x)=1$ for small $|x|$. For $0<\varepsilon \leq 1$ let $\varphi_{\varepsilon}(x)=\varphi(\varepsilon x)$. Then $\left\{\varphi_{\varepsilon}: 0<\varepsilon \leq 1\right\}$ is a uniformly bounded family in $S_{1,0}^{0}$. Let $X_{\varepsilon}=\operatorname{op}\left(\varphi_{\varepsilon}(x) x\right)$. The required identity holds with $x$ replaced by $X_{\varepsilon}$. Moreover, the symbols of $\left[X_{\varepsilon}, A\right]$ form a bounded subset of $S_{\rho, \delta}^{0}$. In view of the fact that $X_{\varepsilon} u \rightarrow x u$ in $\mathcal{S}$ for $u \in \mathcal{S}$ we conclude from Remark 1.2(b) that $\left[X_{\varepsilon}, A\right] v \rightarrow[x, A] v$ in $H_{p}^{-m^{\prime}}$ as $\varepsilon \rightarrow 0^{+}$for $v \in L_{p}$. On the other hand,

$$
\left[X_{\varepsilon},\left(A_{p}-\lambda\right)^{-1}\right] u \rightarrow\left[x,\left(A_{p}-\lambda\right)^{-1}\right] u
$$

in $\mathcal{S}^{\prime}$ whenever $u \in \mathcal{S}$, so we get the desired identity.

Remark 2.8. We shall next deal with the case where $m>\rho$ by using complex powers. Conditions (i) and (ii), or (i) and (ii') respectively, are sufficient for the construction in the following theorem.

Moreover, it is easily checked that the conditions are satisfied for $A-\lambda$ whenever they hold for $A$. We merely have to change the constants $R$ and $c_{0}$ in (ii').

Theorem 2.9. There is a family $\left\{P_{z}: z \in \mathbb{C}\right\}$ of complex powers for $A-\lambda$, satisfying $P_{z} \in \Psi_{\rho, \delta}^{m R e z}, \operatorname{Re} z \geq 0, P_{z} \in \Psi_{\rho, \delta}^{m^{\prime} \operatorname{Re} z}, \operatorname{Re} z<0, P_{z_{1}} P_{z_{2}} \equiv P_{z_{1}+z_{2}}$ modulo $\Psi^{-\infty}, z_{1}, z_{2} \in \mathbb{C}$, and $P_{k} \equiv(A-\lambda)^{k}$ modulo $\Psi^{-\infty}, k \in \mathbb{N}_{0}$.

For a proof see Kumano-go [12, Chapter 8].

2.10. Preparation. Let $\left\{P_{z}: z \in \mathbb{C}\right\}$ be as in Theorem 2.9. Choose an integer $N>m / \rho$ and find suitable $R, R^{\prime}, R^{\prime \prime} \in \Psi^{-\infty}$ so that $A-\lambda=P_{1}+R$ and

$$
\left[x, P_{1}\right]=\left[x, P_{1 / N}^{N}+R^{\prime}\right]=\sum_{k=0}^{N-1} P_{k / N}\left[x, P_{1 / N}\right] P_{(N-1-k) / N}+R^{\prime \prime} .
$$

Proposition 2.11. With the operators $R$ and $P_{k / N}$ as in 2.10 we have

$$
\begin{aligned}
-\left[x,\left(A_{p}-\lambda\right)^{-1}\right]= & \sum_{k=0}^{N-1} P_{k / N-1}\left[x, P_{1 / N}\right] P_{(-1-k) / N} \\
& +\sum_{k=0}^{N-1}\left(A_{p}-\lambda\right)^{-1} S_{1}\left[x, P_{1 / N}\right] P_{(-1-k) / N} \\
& +\sum_{k=0}^{N-1} P_{k / N-1}\left[x, P_{1 / N}\right] S_{2}\left(A_{p}-\lambda\right)^{-1} \\
& +\left(A_{p}-\lambda\right)^{-1}\left(S_{1}\left[x, P_{1 / N}\right] S_{2}+[x, R]\right)\left(A_{p}-\lambda\right)^{-1}
\end{aligned}
$$

in $\mathcal{L}\left(\mathcal{S}, \mathcal{S}^{\prime}\right)$ for suitable operators $S_{1}, S_{2} \in \Psi^{-\infty}$.

Proof. Again choose a function $\varphi \in C_{0}^{\infty}$ with $\varphi(x) \equiv 1$ for small $|x|$, let $\varphi_{\varepsilon}(x)=$ $\varphi(\varepsilon x)$ and, slightly more refined,

$$
X_{\varepsilon, \tilde{\varepsilon}}=\operatorname{op}\left(x \varphi_{\varepsilon}(x) \varphi_{\tilde{\varepsilon}}(\xi)\right), \quad 0<\varepsilon, \tilde{\varepsilon} \leq 1 .
$$


Each of the operators $X_{\varepsilon, \tilde{\varepsilon}}$ is regularizing. We have, with suitable $S_{1}, S_{2} \in \Psi^{-\infty}$,

$$
\begin{aligned}
& -\left[X_{\varepsilon, \tilde{\varepsilon}},\left(A_{p}-\lambda\right)^{-1}\right] \\
= & \left(A_{p}-\lambda\right)^{-1}\left[X_{\varepsilon, \tilde{\varepsilon}}, P_{1}+R\right]\left(A_{p}-\lambda\right)^{-1} \\
= & \sum_{k=0}^{N-1}\left(A_{p}-\lambda\right)^{-1} P_{k / N}\left[X_{\varepsilon, \tilde{\varepsilon}}, P_{1 / N}\right] P_{(N-1-k) / N}\left(A_{p}-\lambda\right)^{-1} \\
& +\left(A_{p}-\lambda\right)^{-1}\left[X_{\varepsilon, \tilde{\varepsilon}}, R\right]\left(A_{p}-\lambda\right)^{-1} \\
= & \sum_{k=0}^{N-1}\left(A_{p}-\lambda\right)^{-1}\left\{(A-\lambda) P_{k / N-1}+S_{1}\right\}\left[X_{\varepsilon, \tilde{\varepsilon}}, P_{1 / N}\right]\left\{P_{(-1-k) / N}(A-\lambda)+S_{2}\right\} \\
& \times\left(A_{p}-\lambda\right)^{-1}+\left(A_{p}-\lambda\right)^{-1}\left[X_{\varepsilon, \tilde{\varepsilon}}, R\right]\left(A_{p}-\lambda\right)^{-1} \\
= & \sum_{k=0}^{N-1} P_{k / N-1}\left[X_{\varepsilon, \tilde{\varepsilon}}, P_{1 / N}\right] P_{(-1-k) / N}+\sum_{k=0}^{N-1}\left(A_{p}-\lambda\right)^{-1} S_{1}\left[X_{\varepsilon, \tilde{\varepsilon}}, P_{1 / N}\right] P_{(-1-k) / N} \\
& +\sum_{k=0}^{N-1} P_{k / N-1}\left[X_{\varepsilon, \tilde{\varepsilon}}, P_{1 / N}\right] S_{2}\left(A_{p}-\lambda\right)^{-1} \\
& +\left(A_{p}-\lambda\right)^{-1}\left(S_{1}\left[X_{\varepsilon, \tilde{\varepsilon}}, P_{1 / N}\right] S_{2}+\left[X_{\varepsilon, \tilde{\varepsilon}}, R\right]\right)\left(A_{p}-\lambda\right)^{-1}
\end{aligned}
$$

for all $0<\varepsilon, \tilde{\varepsilon} \leq 1$ in $\mathcal{L}\left(\mathcal{S}, \mathcal{S}^{\prime}\right)$.

Next fix $\varepsilon>0$ and write, for the moment, $M_{\varepsilon}=$ op $\left(x \varphi_{\varepsilon}(x)\right)$. Clearly, op $\left(\varphi_{\tilde{\varepsilon}}(\xi)\right) u$ $\rightarrow u$ in $\mathcal{S}$ for $u \in \mathcal{S}$ as $\tilde{\varepsilon} \rightarrow 0^{+}$. Hence we obtain convergence

$$
\begin{aligned}
{\left[X_{\varepsilon, \tilde{\varepsilon}}, P_{1 / N}\right] u } & \rightarrow\left[M_{\varepsilon}, P_{1 / N}\right] u \\
{\left[X_{\varepsilon, \tilde{\varepsilon}}, R\right] u } & \rightarrow\left[M_{\varepsilon}, R\right] u
\end{aligned}
$$

in $\mathcal{S}$ for $u \in \mathcal{S}$. The symbols of the commutators $\left\{\left[X_{\varepsilon, \tilde{\varepsilon}}, P_{1 / N}\right]: 0<\tilde{\varepsilon} \leq 1\right\}$ form a bounded subset of $S_{\rho, \delta}^{\mu}$ for $\mu=m / N+\delta-\rho$. Applying 1.2(b), convergence in (2.7) will hold in $H_{p}^{-m^{\prime}-\mu}$ for $u \in L_{p}$. Since $R$ is regularizing, a corresponding argument shows that, for (2.8), convergence is in $L_{p}$ whenever $u \in L_{p}$. On the other hand $\left[X_{\varepsilon, \tilde{\varepsilon}},\left(A_{p}-\lambda\right)^{-1}\right] u \rightarrow\left[M_{\varepsilon},\left(A_{p}-\lambda\right)^{-1}\right] u$ in $\mathcal{S}^{\prime}$ for $u$ in $\mathcal{S}$, so we get equation (2.6) with $x$ replaced by $M_{\varepsilon}$. With analogous considerations as before - in particular the fact that the symbols of the commutators $\left\{\left[M_{\varepsilon}, P_{1 / N}\right]: 0<\varepsilon \leq 1\right\}$ form a bounded subset of $S_{\rho, \delta}^{0}-$ we let $\varepsilon \rightarrow 0^{+}$and obtain the assertion.

Notice that we may not apply the above computation with $X_{\varepsilon, \tilde{\varepsilon}}$ replaced by $x$ : It is not clear, for example, that $\left(A_{p}-\lambda\right)^{-1}\left[x, P_{1}+R\right]\left(A_{p}-\lambda\right)^{-1}$ maps $\mathcal{S}$ to $\mathcal{S}^{\prime}$, even though $\left[X_{\varepsilon, \tilde{\varepsilon}}, P_{1}+R\right] u \rightarrow\left[x, P_{1}+R\right] u$ in $\mathcal{S}$.

Corollary 2.12. $\left[x,\left(A_{p}-\lambda\right)^{-1}\right]: \mathcal{S} \rightarrow \mathcal{S}^{\prime}$ extends to an operator in $\mathcal{L}\left(L_{p}\right)$.

Proof. Each of the four summands on the right-hand side of (2.6) extends to an operator in $\mathcal{L}\left(L_{p}\right)$. Indeed, the first is an element of $\Psi_{\rho, \delta}^{-m^{\prime}}$ hence is $L_{p}$ bounded by Fefferman's theorem. In the other summands, the $L_{p}$-bounded operator $\left(A_{p}-\lambda\right)^{-1}$ is composed with a regularizing pseudodifferential operator.

2.13. Conclusion. In 2.6 we had reduced the problem to showing that, for each multi-index $\alpha$, the operator $\operatorname{ad}^{\alpha} x\left(A_{p}-\lambda\right)^{-1}$ is bounded in $L_{p}$. For $|\alpha|=1$, the assertion has been shown. On the other hand, the identity in Proposition 2.11 
shows that the analysis of higher order commutators reduces to this case. Hence the proof is complete.

Remark 2.14. It is possible to avoid the condition on the argument function in certain other instances. For example suppose that $m-m^{\prime} \leq \rho$. Then we can show, just as is Lemma 2.7, that

$$
\begin{aligned}
{\left[\left(A_{p}-\lambda\right)^{-1}, x\right]=} & B_{\lambda}[x, A] B_{\lambda}+\left(A_{p}-\lambda\right)^{-1} R_{1}[x, A] R_{2}\left(A_{p}-\lambda\right)^{-1} \\
& +B_{\lambda}[x, A] R_{2}\left(A_{p}-\lambda\right)^{-1}+\left(A_{p}-\lambda\right)^{-1} R_{1}[x, A] B_{\lambda} .
\end{aligned}
$$

Hence the first commutator is bounded on $L_{p}$, and so are its iterates, by induction.

\section{ACKNOWLEDGMENT}

The second author would like to thank M. Hieber for valuable discussions on the subject.

\section{REFERENCES}

1. Alvarez, J., and Hounie, J.: Spectral invariance and tameness of pseudo-differential operators on weighted Sobolev spaces, J. Operator Theory 30, 41 - 67 (1993). MR 95j:47062

2. Arendt, W.: Gaussian estimates and interpolation of the spectrum in $L^{p}$, Differential Integral Equations 7, 1153 - 1168 (1993). MR 95e:47066

3. Beals, R.: Characterization of pseudodifferential operators and applications, Duke Math. Journal 44, 5 - 57 (1977). ibid., 46, 215 (1979). MR 55:8884; MR 80b:47062

4. Davies, E. B.: Heat Kernels and Spectral Theory, Cambridge University Press, Cambridge 1989. MR 92a:35035

5. Davies, E. B.: $L^{p}$ spectral independence and $L^{1}$ analyticity, J. London Math. Soc. 52, 177 184 (1995). MR 96e: 47034

6. Davies, E. B., Simon, B., and Taylor, M.: $L^{p}$ spectral theory of Kleinian groups, J. Funct. Anal. 78, 116 - 136 (1988). MR 89m:58205

7. Fefferman, C.: $L^{p}$-bounds for pseudo-differential operators, Israel J. Math. 14, 413-417 (1973). MR 49:1227

8. Gohberg, I., and Krupnik, N.: Einführung in die Theorie der eindimensionalen singulären Integraloperatoren, Birkhäuser, Basel 1979. MR 81d:45010

9. Hempel, R., and Voigt, J.: The spectrum of a Schrödinger operator on $L_{p}\left(\mathbb{R}^{\nu}\right)$ is $p$ independent, Comm. Math. Phys. 104, 243 - 250 (1986). MR 87h:35247

10. Hieber, M.: $L^{p}$ spectra of pseudodifferential operators generating integrated semigroups, Transactions Am. Math. Soc. 347, 4023 - 4035 (1995). MR 95m:47099

11. Illner, R.: On algebras of pseudodifferential operators in $L^{p}$, Comm. Partial Diff. Equations 2, 359 - 393 (1977). MR 56:1137b

12. Kumano-go, H.: Pseudo-Differential Operators, Massachusetts Institute of Technology Press, Cambridge (Massachusetts)-London 1981. MR 84c:35113

13. Kumano-go, H., and Tsutsumi, C.: Complex powers of hypoelliptic pseudo-differential operators with applications, Osaka Journal Math. 10, 147 - 174 (1973). MR 48:6734

14. Leopold, H.-G., and Schrohe, E.: Spectral invariance for algebras of pseudodifferential operators on Besov-Triebel-Lizorkin spaces, manuscripta math. 78, 99 - 110 (1993). MR 93m:47061

15. Schechter, M.: Spectra of Partial Differential Operators, 2.ed. North-Holland, Amsterdam, New York, Oxford 1986. MR 88h:35085

16. Schrohe, E.: Boundedness and spectral invariance for standard pseudodifferential operators on anisotropically weighted $L^{p}$-Sobolev spaces, Integral Equations Operator Theory 13, 271 284 (1990). MR 91b:47121

17. Schrohe, E.: Fréchet Algebras of Pseudodifferential Operators and Boundary Value Problems, Birkhäuser, Basel (to appear).

18. Sturm, K.-Th.: On the $L^{p}$ spectrum of uniformly elliptic operators on Riemannian manifolds, J. Funct. Anal. 118, 442 - 453 (1993). MR 94m:58227

19. Ueberberg, J.: Zur Spektralinvarianz von Algebren von Pseudodifferentialoperatoren in der $L^{p}$-Theorie, manuscripta math. 61, 459 - 475 (1988). MR 89g:47070 
20. Widom, H.: Singular integral equations in $L^{p}$, Transactions Am. Math. Soc. 97, 131 - 160 (1960). MR 22:9830

21. Wong, M.W.: Spectra of pseudo-differential operators on $L^{p}\left(R^{n}\right)$, Comm. Partial Diff. Equations 4, 1389 - 1401 (1979). MR 81a:47052

Mathematisches Institut, Fakultät FÜr Mathematik Und Informatik, FriedrichSchiller-Universität Jena, D-07740 Jena, Germany

E-mail address: leopold@minet.uni-jena.de

Max-Planck-Arbeitsgruppe "Partielle Differentialgleichungen und Komplexe AnaLysis", Universität Potsdam, D-14415 Potsdam, Germany

E-mail address: schrohe@mpg-ana.uni-potsdam.de 